\title{
Discovery of a widely separated binary system of very low mass stars ${ }^{\star}$
}

\author{
N. Phan-Bao ${ }^{1}$, E. L. Martín ${ }^{2,3}$, C. Reylé ${ }^{4}$, T. Forveille ${ }^{5,6}$, and J. Lim ${ }^{1}$ \\ ${ }^{1}$ Institute of Astronomy and Astrophysics, Academia Sinica, PO Box 23-141, Taipei 106, ROC, Taiwan \\ e-mail: pbngoc@asiaa.sinica.edu.tw \\ 2 Instituto de Astrofísica de Canarias, C/ vía Láctea s/n, 38200 La Laguna (Tenerife), Spain \\ ${ }^{3}$ University of Central Florida, Dept. of Physics, PO Box 162385, Orlando, FL 32816-2385, USA \\ 4 CNRS UMR6091, Observatoire de Besançon, BP 1615, 25010 Besançon Cedex, France \\ 5 Canada-France-Hawaii Telescope Corporation, 65-1238 Mamalahoa Highway, Kamuela, HI 96743, USA \\ ${ }^{6}$ Laboratoire d'Astrophysique de Grenoble, Université J. Fourier, BP 53, 38041 Grenoble, France
}

Received 3 June 2005 / Accepted 3 July 2005

\begin{abstract}
We report our discovery of a nearby wide binary system, LP 714-37 AB. Phan-Bao et al. identified LP 714-37 as a mid-M dwarf during a cross-identification of the NLTT and DENIS catalogues. Our CCD images resolve the system into a binary with a projected angular separation of $1.8^{\prime \prime}$, or $33 \mathrm{AU}$, and low-resolution optical spectra give spectral types of M5.5 (LP 714-37A) and M7.5 (LP 714-37B). This makes LP 714-37 AB one of very few widely separated (separation $>30$ AU) very low-mass binary systems known in the field, and we discuss it in the context of the multiplicity properties of very low-mass stars and brown dwarfs.
\end{abstract}

Key words. binary stars - very low mass stars, brown dwarfs - individual star: DENIS-P J0410-1251, LP 714-37

\section{Introduction}

Binary systems have been studied for decades to measure accurate stellar masses, and to test evolutionary models and star formation theories. Considerable attention has recently been paid to very low-mass (VLM) companions to low-mass stars (Duquennoy \& Mayor 1991; Fischer \& Marcy 1992; Delfosse et al. 1999; Reid et al. 2001; Beuzit et al. 2004; Forveille et al. 2004), as well as to binaries among ultracool dwarfs (spectral types later than M6) in the solar neighborhood (Martín et al. 1999a; Close et al. 2002, 2003; Bouy et al. 2003; Burgasser et al. 2003; Gizis et al. 2003; Siegler et al. 2005; Forveille et al. 2005) and in nearby young open clusters and associations (Martín et al. 1998, 2000, 2003; Chauvin et al. 2004). The main results of the high spatial resolution imaging surveys of VLM stars and brown dwarfs (BDs) can be summarized as follows: (1) the binary frequency in the separation range 1-15 AU is about $15 \%$; (2) the frequency of wide binary systems (semimajor axis $>15 \mathrm{AU}$ ) is very low, $<1 \%$; (3) the mass ratios are strongly biased towards nearly-equal mass binaries, beyond the expected observation selection effects. The companions to the more massive low mass stars, by contrast, span a wider range of both separations and mass ratios.

The properties of VLM binaries are an important constraint for models of star-formation and evolution. It has been

* Based on observations made at the European Southern Observatory, La Silla, Chile. debated in the literature whether the properties of VLM binaries and stellar binaries differ, implying different formation mechanisms (Kroupa et al. 2003), or whether the binary properties instead show continuous trends with decreasing primary mass, implying that VLM binaries form through the same processes as stellar binaries (Luhman 2004b). Clearly there is a need for a larger sample of observed VLM binaries, particularly at wide separations where few of them are known. One leading model of $\mathrm{BD}$ formation is that they form and are ejected in unstable multiple systems within small clusters (Bate et al. $2002,2003)$. Numerical simulations of decaying $N$-body clusters indicate that the typical separations of binaries composed of stars with masses ranging between $0.1 M_{\odot}$ and $0.2 M_{\odot}$ would be below 10 AU (Sterzik \& Durisen 1998), in rough agreement with the observations. The ejection models (Reipurth \& Clarke 2001; Bate et al. 2002) suggest that the binary BD systems that do exist must be close (separations $\leq 10 \mathrm{AU}$ ). The detection of wide VLM binary systems has thus become an important test of the ejection models. The first wide binary BDs have been found in young ( $<10 \mathrm{Myr})$ associations or clusters: 2MASS J1101-7732 (240 AU separation, in Chamaeleon I, Luhman 2004a); 2MASS J1207-3932 (55 AU, in TW Hydrae, Chauvin et al. 2004). Their existence is at first sight inconsistent with the ejection models, though the statistics of the numerical simulations is currently limited.

In this Letter, we present a new wide binary consisting of two VLM stars in the field. Section 2 presents the observations 


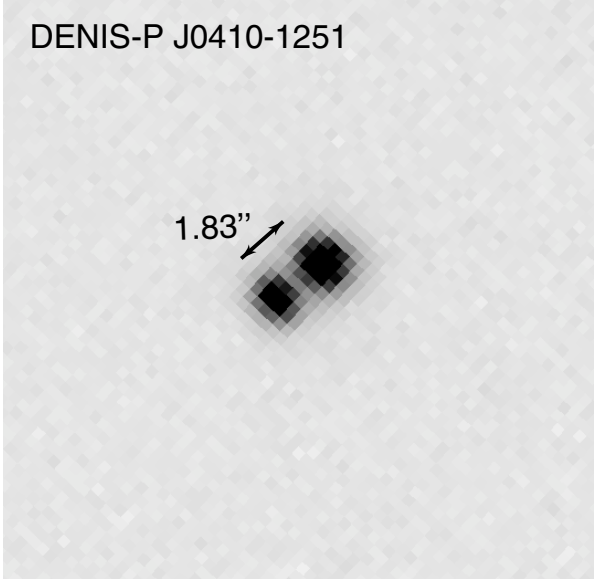

Fig. 1. A $20 \times 20^{\prime \prime}$ section of the $R$-band NTT image of the LP 714-37 binary system. The pixel size is $0.33^{\prime \prime}$, North is up and East is left.

and data reduction, while Sect. 3 discusses the results in the context of the binary properties of VLM stars and BDs.

\section{Observations and data reduction}

Phan-Bao et al. (2003) identified LP 714-37 (DENISP J0410-1251) as a mid-M dwarf while cross-identifying the NLTT (Luyten 1980) and DENIS (Epchtein 1997) catalogues, and derived a photometric distance of $15.3 \mathrm{pc}$. Cruz et al. (2003) independently determined a spectrophotometric distance of $15.4 \mathrm{pc}$ and an M6.0 spectral type. Allowing for the binary nature of the system pushes its distance out to $18.1 \mathrm{pc}$ (Sect. 3).

As part of our ongoing spectroscopic follow-up of new nearby $\mathrm{M}$ dwarfs detected with DENIS, one of us (Céline Reylé) observed LP 714-37 with the 3.6-m New Technology Telescope (NTT) at La Silla Observatory (ESO, Chile) in November 2003; and the data was analyzed in January 2005. Two objects were in fact detected on the acquisition image (Fig. 1).

Optical low-resolution spectra were obtained for both objects in the Red Imaging and Low-dispersion spectroscopy (RILD) observing mode of the EMMI instrument. The detector was a $2048 \times 4096 \mathrm{MIT} / \mathrm{LL}$ CCD, used in normal readout mode with $2 \times 2$ binning. Grism\#2 gave a dipersion of $3.5 \AA$ pixel $^{-1}$, and the OG530\#645 order sorting filter defined a wavelength range of 520 to $950 \mathrm{~nm}$. The slit width of 1 arcsec corresponds to a spectral resolution of $10.4 \AA$, and the seeing was 0.7 arcsec. The exposure time was 30 s. LTT 2415 and Feige 110 (selected from the ESO list, ftp.eso.org/pub/stecf/standards/ctiostan/) were observed as spectrophotometric standards. The reduction of spectra was performed with MIDAS packages. We normalized the spectra to 1 over the $754-758 \mathrm{~nm}$ interval, the denominator of the PC3 index (Martín et al. 1999b) and a region with a good flat pseudo-continuum. Figure 2 shows the two resulting spectra. The presence of the NaI and KI doublets and the absence of the CaII triplet immediately demonstrate that both stars are $\mathrm{M}$ dwarfs rather than $\mathrm{M}$ giants, as does the general shape of the $\mathrm{TiO}$ bands. Analysis of the $R$ band acquisition

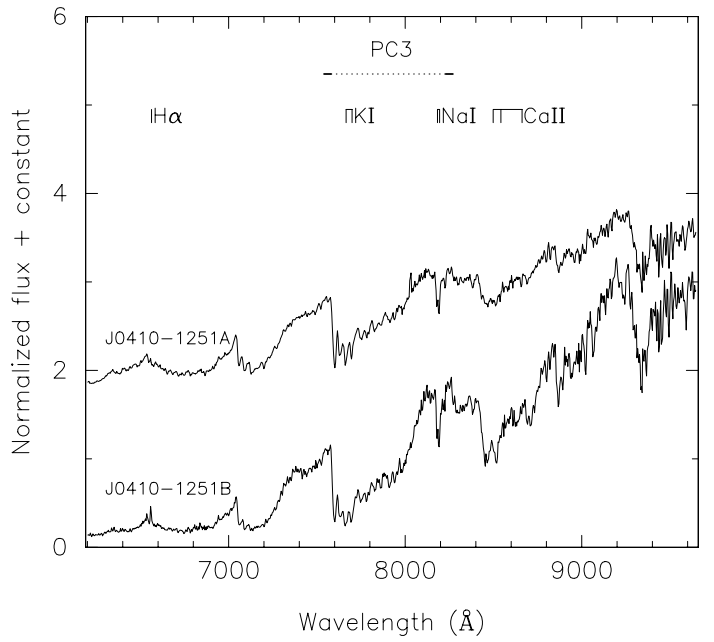

Fig. 2. Spectra of the two components of LP 714-37. The positions of the $\mathrm{H}_{\alpha}, \mathrm{NaI}, \mathrm{KI}$ and CaII lines are indicated, as well as the spectral intervals used to compute the PC3 spectral index.

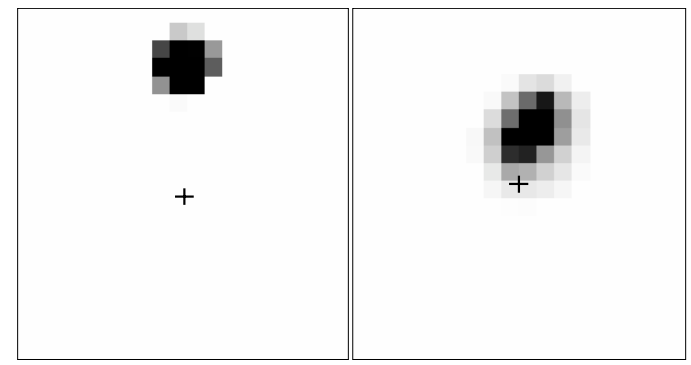

Fig. 3. Archival images of LP 714-37: SERC-I (left, epoch: 1986.940) and DENIS-I (right, epoch: 2000.896). The cross indicates the position of component B at the 2003.915 epoch of the NTT image. Clearly, the SERC-I image would easily separate the two objects if they were not physically associated. The size of each image is $20 \times 20^{\prime \prime}$, and North is up and East to the left.

image with SExtractor (Bertin \& Arnouts 1996) gives a separation of 5.53 pixels, or $1.83^{\prime \prime}$ with the $0.33^{\prime \prime} /$ pixel focal scale of EMMI. Since the discovery is serendipitous we did not obtain images with other filters and have no photometric calibrations for the $R$ band. We therefore only use the image for astrometry.

\section{Discussion}

The calibration of the PC3 index to spectral type (Martín et al. 1999b) gives spectral types of M5.5 for component A and M7.5 for component B, with an uncertainty of \pm 0.5 subclass. The observed $\mathrm{H}_{\alpha}$ emission in component $\mathrm{B}$ with spectral type of M7.5 is consistent with the observations by Gizis et al. (2000a) pointed out that all M7-M8 dwarfs show the $\mathrm{H}_{\alpha}$ line in emission.

The proper motion of the system is $\mu_{\alpha}=-117 \mathrm{mas} / \mathrm{yr}$ and $\mu_{\delta}=-382 \mathrm{mas} / \mathrm{yr}$ (Phan-Bao et al. 2003), and it has therefore moved by 6.8 arcsec between the epoch of the SERC-I plate and our NTT observation. Figure 3 shows that there is no background star at the position of the system in either the SERC-I 
Table 1. PC3 indices and spectral types of the two components of LP 714-37.

\begin{tabular}{|c|c|c|c|c|c|c|c|c|c|c|c|c|}
\hline $\begin{array}{l}\text { Stars } \\
\text { (1) }\end{array}$ & $\begin{array}{l}\text { Other name } \\
\text { (2) }\end{array}$ & $\begin{array}{l}\text { PC3 } \\
\text { (3) }\end{array}$ & $\begin{array}{l}\mathrm{SpT} \\
\text { (4) }\end{array}$ & $\begin{array}{l}M_{I} \\
\text { (5) }\end{array}$ & $\begin{array}{l}M_{J} \\
(6)\end{array}$ & $\begin{array}{l}M_{H} \\
\text { (7) }\end{array}$ & $\begin{array}{l}M_{K} \\
(8)\end{array}$ & $\begin{array}{l}\text { Mass } \\
\left(M_{\odot}\right) \\
(9)\end{array}$ & $\begin{array}{l}\text { Sep. } \\
(\mathrm{AU}) \\
(10)\end{array}$ & $\begin{array}{l}P \\
(\mathrm{yr}) \\
(11)\end{array}$ & $\begin{array}{l}\text { Dist. } \\
\text { (pc) } \\
(12)\end{array}$ & $\begin{array}{l}\mu \\
" / \mathrm{yr} \\
(13)\end{array}$ \\
\hline LP 714-37A & DENIS-P J0410-1251 & 1.32 & M5.5 & 11.96 & 9.95 & 9.34 & 9.11 & 0.11 & $33.1 \pm 4.0$ & 426 & $18.1 \pm 2.2$ & 0.400 \\
\hline LP 714-37B & & 1.72 & M7.5 & 13.56 & 10.85 & 10.24 & 9.82 & 0.09 & & & & \\
\hline
\end{tabular}

Columns 1 and 2: NLTT and DENIS name; Cols. 3 and 4: the PC3 index and spectral types derived from the (PC3, spectral type) relation of Martín et al. (1999b); Cols. 5-8: absolute magnitudes for the $I, J, H$, and $K$ bands based on the PC3-absolute magnitudes relation (Crifo et al. 2005); Col. 9: mass determinations for 1-5 Gyr from the models of Baraffe et al. (1998); Col. 10: projected separation; Col. 11: period computed by assuming a face-on circular orbit; Cols. 12: spectrophotometric distance; Cols. 13: total proper motion taken from Phan-Bao et al. (2003).

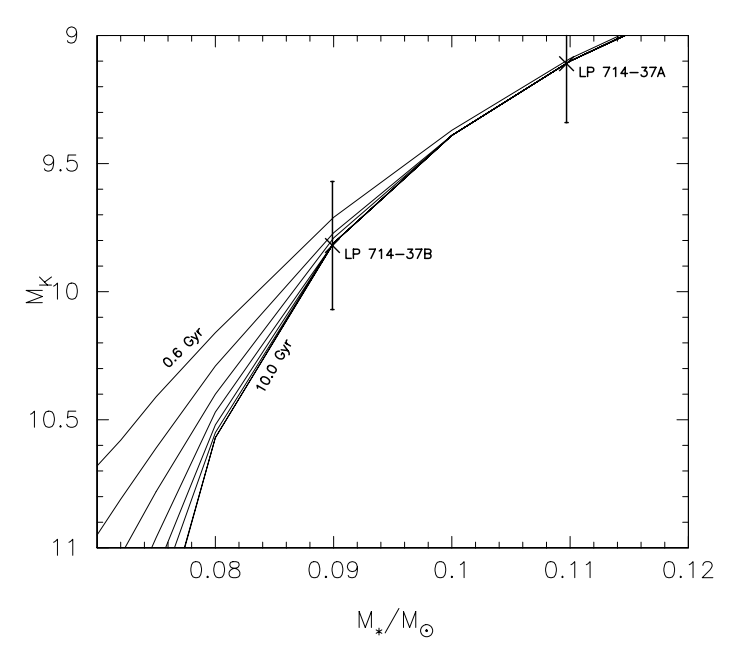

Fig. 4. The mass vs. $K$ band absolute magnitude relations from the evolutionary models of Baraffe et al. (1998) for solar metallicity low mass stars. The crosses indicate the masses of components A and B for an age of $5 \mathrm{Gyr}$. The isochrones plotted are $0.6,0.8,1.0,1.3,1.6$, 2.0, 5.0, and $10.0 \mathrm{Gyr}$; the latter two are overlapped.

image ( $I$ band) or the DENIS-I image, and therefore demonstrates that the system is a physical binary.

To estimate the distance, we assume that this system has no additional unresolved component(s). We can then calculate the absolute magnitudes of the system from those of the two components, computed from their PC3 indices with the PC3 index to magnitudes relation of Crifo et al. (2005) (Table 1). Comparison with the DENIS apparent magnitudes $(I=12.99, J=10.94, K=9.89)$ then gives the distance: $d_{I}=17.8 \pm 2.1 \mathrm{pc} ; d_{J}=18.9 \pm 2.3 \mathrm{pc}$ and $d_{K}=17.6 \pm 2.1 \mathrm{pc}$. We adopt the mean distance of $d=18.1 \pm 2.2 \mathrm{pc}$.

We estimate the mass of each component, using $I, J$, and $K$-band mass-luminosity relations (Fig. 4 for the $K$ band) from the Baraffe et al. (1998) models. We adopt their 5 Gyr model, an intermediate age for the solar neighbourhood (Caloi et al. 1999), but the results are insensitive to this choice (Fig. 4). The masses of component $\mathrm{A}$ and $\mathrm{B}$ are respectively $0.11 \pm 0.01 M_{\odot}$ and $0.09 \pm 0.005 M_{\odot}$, slightly above the hydrogen-burning limit (Chabrier \& Baraffe 1997). The total mass of LP 714-37AB is $0.2 \pm 0.015 M_{\odot}$, within one sigma of the $M_{\text {tot }}<0.185 M_{\odot}$ convention adopted by Close et al. (2002) for their sample of VLM binaries. Thus, this system can be considered a VLM binary. At the $18.1 \mathrm{pc}$ of the system, its $1.83^{\prime \prime}$ separation corresponds to

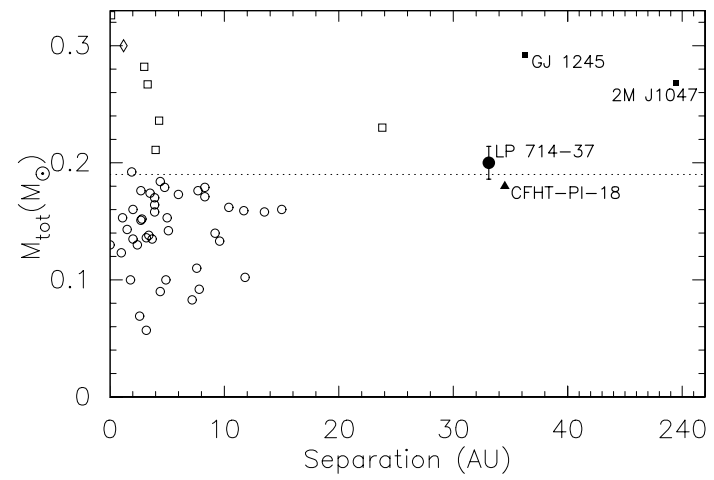

Fig. 5. Sum of primary and secondary component masses vs. projected binary separation for VLM binaries in the field. The empty circles represent binaries with separations smaller than $30 \mathrm{AU}$, from Table 4 of Siegler et al. (2005) and references therein. The empty squares show low mass field binaries from Reid \& Gizis (1997) with masses updated from RECONS at http://www . chara.gsu. edu/RECONS/. The empty rhombus is LHS 224 from Beuzit et al. (2004). The 35 AU VLM binary CFHT-Pl-18 (Martín et al. 2000) is plotted as a filled triangle. The filled squares show two VLM triple systems GJ 1245 and 2MASS J1047+4026, discussed in Sect. 3. The filled circle indicates LP 714-37. Dotted line: $M_{\text {tot }}=0.185 M_{\odot}$, the limit for VLM systems adopted by Close et al. (2003).

33.1 AU. Assuming a face-on circular orbit, the corresponding orbital period is approximately 400 years.

Recent surveys demonstrate that VLM binaries with large separations (>30 AU) are rare in the field (Fig. 5, and references therein), but can be found in young associations and clusters (Chauvin et al. 2004; Luhman 2004a). Those authors reported the respective discoveries of 2MASSW J1207-3932 (8 Myr, TW Hydrae), and 2MASSW J1101-7732 (1 Myr, Chamaeleon I) with separations of 55 AU and $240 \mathrm{AU}$. We report here the discovery of a 33 AU VLM binary, and Martín et al. (2000) found that CFHT-Pl-18 is a 35 AU VLM binary (Fig. 5), both in the field.

Since ejection models predict a maximum separation of about 10 AU for VLM binaries, the existence of the wide binaries is at first sight inconsistent with these ejection models. One should note however that the numerical models to date suffer from small number statistics. A further caveat if that the relevant quantity is the total mass of the system, and that the apparent binaries could possibly be triple or higher order multiple systems, with a correspondingly higher total mass. 
This would make them analogs of the GJ $1245 \mathrm{ABC}$ triple system, which consists of two M5.5 and one $\sim$ M8 dwarfs (Reid et al. 1995; Henry et al. 1999) with separations of 32 and 5 AUs (McCarthy et al. 1988). We note that Gizis et al. (2000b) discovered a 230 AU VLM binary in the field, LP 213-67 (M6.5) and LP 213-68 (M8.0), which in the recent adaptive optics survey of Close et al. (2003) turned out to be triple when LP 213-67AB (or 2MASS J1047+4026) was resolved into two components (see Fig. 5). Bouy et al. (2005) further reported that the DENIS-P J020529.0-115925 VLM binary is a probable triple system. Triple systems could thus potentially explain the apparent excess of wide VLM binaries, and adaptive optics imaging of LP 714-37 would be of obvious interest to clarify its true multiplicity.

Amongst the recently discovered VLM field binaries (Bouy et al. 2003; Close et al. 2002, 2003; Siegler et al. 2005; Forveille et al. 2005), LP 714-37 has one of the widest separation, making it of great interest as a constraint for VLM binary star formation theories. We note that late-M dwarfs detected by Phan-Bao et al. (2001, 2003) over 5700 square degrees in the DENIS database provide a valuable well defined sample for studies of VLM field binaries.

Acknowledgements. Ngoc Phan-Bao is grateful to the DENIS consortium for access to the DENIS data used by his very low mass stars search. Ngoc Phan-Bao also thanks Françoise Crifo for her comments on the manuscript. Celine Reylé acknowledges help during the observations by Olivier Hainaut and the NTT team at the European Southern Observatory. We thank the referee, Kevin Luhman, for a fast and useful report. Partial funding was provided by NSF grant AST 02-05862.

\section{References}

Baraffe, I., Chabrier, G., Allard, F., \& Hauschildt, P. H. 1998, A\&A, 337,403

Bate, M. R., Bonnell, I. A., \& Bromm, V. 2002, MNRAS, 332, L65

Bate, M. R., Bonnell, I. A., \& Bromm, V. 2003, MNRAS, 339, 577

Bertin, E., \& Arnouts, S. 1996, A\&AS, 117, 393

Beuzit, J.-L., Ségransan, D., Forveille, T., et al. 2004, A\&A, 425, 997

Bouy, H., Brandner, W., Martín, E. L., et al. 2003, AJ, 126, 1526

Bouy, H., Martín, E. L., Brandner, W., \& Bouvier, J., 2005, AJ, 129, 511

Burgasser, A. J., Kirkpatrick, J. D., Reid, I. N., et al. 2003, ApJ, 586, 512
Caloi, V., Cardini, D., D’Antona, F., et al. 1999, A\&A, 351, 925

Chabrier, G., \& Baraffe, I. 1997, A\&A, 327, 1039

Chauvin, G., Lagrange, A.-M., Dumas, C., et al. 2004, A\&A, 425, L29

Close, L. M., Siegler, N., Potter, D., Brandner, W., Liebert, J., et al. 2002, ApJ, 567, L53

Close, L. M., Siegler, N., Freed, M., \& Biller, B. 2003, ApJ, 587, 407

Crifo, F., Phan-Bao, N., Delfosse, X., et al. 2005, A\&A, accepted

Cruz, K. L., Reid, I. N., Liebert, J., Kirkpatrick, J. D., \& Lowrance, P. J. 2003, AJ, 126, 2421

Delfosse, X., Forveille, T., Beuzit, J.-L., et al. 1999, A\&A, 344, 897

Duquennoy, A., \& Mayor, M. 1991, A\&A, 248, 485

Epchtein, N. 1997, in the 2nd DENIS Euroconference, The impact of large scale near-infrared surveys, ed. F. Garzon, et al. (Dordrecht: Kluwer), 15

Fischer, D. A., \& Marcy, G. W. 1992, ApJ, 396, 178

Forveille, T., Ségransan, D., Delorme, P., et al. 2004, A\&A, 427, L1

Forveille, T., Beuzit, J.-L., Delorme, P., et al. 2005, A\&A, L435

Gizis, J. E., Monet, D. G., Reid, I. N., et al. 2000a, AJ, 120, 1085

Gizis, J. E., Monet, D. G., Reid, I. N., Kirkpatrick, J. D., \& Burgasser, A. J. 2000b, MNRAS, 311, 385

Gizis, J. E., Reid, I. N., Knapp, G. R., et al. 2003, AJ, 125, 3302

Henry, T. J., Franz, O. G., Wasserman, L. H., et al. 1999, ApJ, 512, 864

Kroupa, P., Bouvier, J., Duchêne, G., \& Moraux, E. 2003, MNRAS, 346,354

Luhman, K. L. 2004a, ApJ, 614, 398

Luhman, K. L. 2004b, ApJ, 617, 1216

Luyten, W. J. 1980, New Luyten catalog of stars with proper motions larger than Two Tenths of an arcsecond, NLTT, Minneapolis, University of Minnesota

Martín, E. L., Basri, G., Brandner, W., et al. 1998, ApJ, 509, L113

Martín, E. L., Brandner, W., \& Basri, G. 1999a, Science, 283, 1718

Martín, E. L., Delfosse, X., Basri, G., et al. 1999b, AJ, 118, 2466

Martín, E. L., Brandner, W., Bouvier, J., et al. 2000, ApJ, 543, 299

Martín, E. L., Barrado y Navascués, D., Baraffe, I., Bouy, H., \& Dahm, S. 2003, ApJ, 594, 525

McCarthy, D. W., Jr., Henry, T. J., et al. 1988, AJ, 333, 943

Phan-Bao, N., Guibert, J., Crifo, F., et al. 2001, A\&A, 380, 590

Phan-Bao, N., Crifo, F., Delfosse, X., et al. 2003, A\&A, 401, 959

Reid, I. N., Hawley, S. L., \& Gizis, J. E. 1995, AJ, 110, 1838

Reid, I. N., \& Gizis, J. E. 1997, AJ, 113, 2246

Reid, I. N., Gizis, J. E., Kirkpatrick, J. D., \& Koerner, D. W. 2001, AJ, 121,489

Reipurth, B., \& Clarke, C. 2001, AJ, 122, 432

Siegler, N., Close, L. M., Cruz, K. L., Martín, E. L., \& Reid, I. N. 2005, ApJ, 621, 1023

Sterzik, M. F., \& Durisen, R. H. 1998, A\&A, 339, 95 